\title{
The precise boundary trace of solutions of a class of supercritical nonlinear equations
}

\author{
Moshe Marcus ${ }^{\mathrm{a}}$, Laurent Véron ${ }^{\mathrm{b}}$

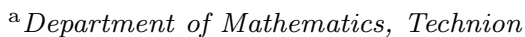 \\ Haifa 32000, ISRAEL \\ ${ }^{\mathrm{b}}$ Laboratoire de Mathématiques et Physique Théorique, Faculté des Sciences \\ Parc de Grandmont, 37200 Tours, FRANCE
}

\begin{abstract}
We construct and study the properties of the precise boundary trace of positive solutions of $-\Delta u+u^{q}=0$ in a smooth bounded domain of $\mathbb{R}^{N}$, in the supercritical case $q \geq q_{c}=(N+1) /(N-1)$. To cite this article: $M$. Marcus, L. Véron, C. R. Acad. Sci. Paris, Ser. I XXX (2006).

\section{Résumé}

La trace au bord précise des solutions d'une classe d'équations non linéaires sur-critiques Nous construisons et étudions les propriétés de la trace au bord précise des solutions positives de $-\Delta u+u^{q}=0$ dans un domaine régulier de $\mathbb{R}^{N}$, dans le cas sur-critique $q \geq q_{c}=(N+1) /(N-1)$. Pour citer cet article : M. Marcus, L. Véron, C. R. Acad. Sci. Paris, Ser. I XXX (2006).
\end{abstract}

Version française abrégée Soit $\Omega$ un ouvert borné de $\mathbb{R}^{N}$ de bord de classe $C^{2}$ et $u \in L_{l o c}^{q}(\Omega)(q>1)$ une solution positive de

$$
-\Delta u+|u|^{q-1} u=0 .
$$

Il est bien connu que $u$ possède une trace au bord dans la classe des mesures de Borel ayant la régularité extérieure au sens classique. Si $q \geq q_{c}$ cette notion de trace n'est pas suffisante pour déterminer de façon unique la solution de (1). Contrairement à la trace fine [5], qui s'exprime en termes probabilistes et est limitée au cas $q_{c} \leq q \leq 2$, la notion de trace précise que nous développons est valable pour tout $q \geq q_{c}$. Elle est fondée sur la topologie fine $\mathfrak{T}_{q}$ associée à la capacité de Bessel $C_{2 / q, q^{\prime}}$ sur $\partial \Omega$. Notons

\footnotetext{
Email addresses: marcusm@math.technion.ac.il (Moshe Marcus), veronl@univ-tours.fr (Laurent Véron).
} 
$\rho(x):=\operatorname{dist}(x, \partial \Omega)$ et $\Omega_{\beta}=\{x \in \Omega: \rho(x)<\beta\}, \quad \Omega_{\beta}^{\prime}=\omega \backslash \bar{\Omega}_{\beta}, \quad \Sigma_{\beta}=\partial \Omega_{\beta}^{\prime}$. Il existe $\beta_{0}>0$ tel que pour tout $x \in \Omega_{\beta_{0}}$ il existe un unique $\sigma(x) \in \Omega$ tel que $\rho(x)=|x-\sigma(x)|$. Si $Q \subset \partial \Omega$ est $\mathfrak{T}_{q}$-ouvert, on note $\Sigma_{\beta}(Q)=\left\{x \in \Sigma_{\beta}: \sigma(x) \in Q\right\}$, et, si $u \in C(\Omega)$, $u_{\beta}^{Q}$ désigne la solution de (1) dans $\Omega_{\beta}^{\prime}$ valant $u \chi_{\Sigma_{\beta}(Q)}$ sur $\Sigma_{\beta}$. La dichotomie suivante est à la base de nos résultats.

Théorème 1 Soit u une solution positive de (1). Si $Q \subset \partial \Omega$ est $\mathfrak{T}_{q}$-ouvert et si la limite suivante existe, on pose $L(Q)=\lim _{\beta \rightarrow 0} \int_{\Sigma_{\beta}(Q)} u d S$. Alors,

ou bien, $L(Q)=\infty$ pour tout voisinage $\mathfrak{T}_{q}$-ouvert $Q$ de $\xi$,

ou bien, il existe un voisinage $\mathfrak{T}_{q}$-ouvert $Q$ de $\xi$ tel que $L(Q)<\infty$.

Le premier cas se produit si et seulement si, pour tout $\mathfrak{T}_{q}$ - voisinage $Q$ de $\xi$,

$$
\int_{A} u^{q} \rho(x) d x=\infty, \quad A=\left(0, \beta_{0}\right) \times Q .
$$

Un point $\xi$ est dit singulier (resp. régulier) si le premier (resp. le deuxième) cas se produit. L'ensemble des point singuliers (resp. réguliers) noté $\mathcal{S}(u)$ (resp. $\mathcal{R}(u)$ est $\mathfrak{T}_{q}$-fermé (resp. $\mathfrak{T}_{q}$-ouvert). Si $A \subset \partial \Omega$, nous notons $\tilde{A}$ la fermeture de $A$ dans topologie $\mathfrak{T}_{q}$.

Théorème $\mathbf{2}$ Il existe une mesure de Borel positive $\mu$ sur $\partial \Omega$ possédant les propriétés suivantes :

(i) Pour tout $\sigma \in \mathcal{R}(u)$ il existe un voisinage $\mathfrak{T}_{q}$-ouvert $Q$ de $\sigma$ et une solution modérée $w$ de (1) tels que $\tilde{Q} \subset \mathcal{R}(u), \mu(\tilde{Q})<\infty$ et

$$
u_{\beta}^{Q} \rightarrow w \text { localement uniformément dans } \Omega, \quad(\operatorname{tr} w) \chi_{Q}=\mu \chi_{Q} .
$$

(ii) $\quad \mu$ a la régularité extérieure pour la topologie $\mathfrak{T}_{q}$ et est absolument continue par rapport à la capacité $C_{2 / q, q^{\prime}}$ sur les sous-ensembles $\mathfrak{T}_{q}$-ouverts où elle est bornée.

Le couple $(\mu, \mathcal{S}(u)$ est, par définition, la trace précise de $u$, notée $\operatorname{tr}(u)$, qui peut être aussi représentée par la mesure de Borel $\nu$ définie par $\nu=\mu \operatorname{sur} \mathcal{R}(u)$ et $\nu(A)=\infty$ pour tout borélien $A$ tel que $A \cap \mathcal{S}(u) \neq \emptyset$; $\nu$ a les propriétés suivantes : (i) Elle a la régularité extérieure pour la topologie $\mathfrak{T}_{q}$. (ii) Elle est absolument continue par rapport à la capacité $C_{2 / q, q^{\prime}}$ au sens où pour tout ensemble $\mathfrak{T}_{q}$-ouvert $Q$ et tout borélien $A, C_{2 / q, q^{\prime}}(A)=0$ implique $\nu(Q)=\nu(Q \backslash A)$. Une mesure de Borel vérifiant (i) et (ii) est dite q-parfaite. Nous donnons alors la condition nécessaire et suffisante d'existence, ainsi qu'un résultat d'unicité, de la solution du problème aux limites généralisé

$$
-\Delta u+u^{q}=0, \quad u>0 \text { dans } \Omega, \quad \operatorname{tr}(u)=\nu,
$$

Théorème 3 Soit $\nu$ une mesure de Borel sur $\partial \Omega$, bornée ou non. Le problème aux limites (4) a une solution si et seulement si $\nu$ est q-parfaite. Quand c'est le cas, une solution de (4) est donnée par

$$
U=v \oplus U_{F}, \quad v=\sup \left\{u_{\nu \chi_{Q}}: Q \in \mathcal{F}_{\nu}\right\}
$$

où $\mathcal{F}_{\nu}:=\{Q: Q$ q-ouvert, $\nu(Q)<\infty\}, G:=\bigcup_{\mathcal{F}_{\nu}} Q, F=\partial \Omega \backslash G, U_{F}$ est la solution maximale s'annullant sur $\partial \Omega \backslash F$ et $v \oplus U_{F}$ est la plus grande solution de (1) inférieure à la sur-solution $v+U_{F}$. Enfin $U$ est $\sigma$-modérée, c'est la solution maximale du problème (4) dont c'est l'unique solution $\sigma$-modérée.

\section{Introduction and statement of results}

In this paper we present a theory of boundary trace of positive solutions of the equation

$$
-\Delta u+|u|^{q-1} u=0
$$


in a bounded domain $\Omega \subset \mathbb{R}^{N}$ of class $C^{2}$. A function $u$ is a solution if $u \in L_{l o c}^{q}(\Omega)$ and the equation holds in the distribution sense.

Equations of this type have been intensively studied in the last ten years in the context of boundary trace theory and the associated boundary value problem. In the subcritical case, $1<q<q_{c}=(N+1) /(N-1)$, the problem is well understood thanks to the works of Le Gall [7], using a probabilistic approach which imposes $q \leq 2$, and Marcus and Véron [9] by using an analytic approach, with no restriction. In the supercritical case $q \geq q_{c}$, the notions of removable sets and admissible Radon measures are implemented by Le Gall, by Dynkin and Kuznetsov [4] and by Marcus and Véron [10,11]. But in 1997 Le Gall showed that the standard trace theory is not appropriate because many solutions may have the same boundary trace. Following this observation, a theory of fine trace, based upon a probabilistic formulation, was introduced by Dynkin and Kuznetsov [5] who showed that, for $q \leq 2$, the fine trace theory is satisfactory in the family of so-called $\sigma$-moderate solutions. Later on it was shown by Mselati [13], combining Le Gall's Brownian snake method [8] and Dynkin's approach [2] that, in the case $q=2$, all positive solutions are $\sigma$-moderate. By analytical methods Marcus and Véron [12] proved that, for all $q \geq q_{c}$ and every compact set $K \subset \partial \Omega$, the maximal solution of (6) vanishing on $\partial \Omega \backslash K$ is $\sigma$-moderate. Their proof was based on the derivation of sharp capacitary estimates for the maximal solution. In continuation, Dynkin [3], using the estimates of Marcus and Veron [12], extended Mselati's result to all case $q \leq 2$. For $q>2$ the problem remains open.

Our definition of boundary trace is based on the fine topology associated with the Bessel capacity $C_{2 / q, q^{\prime}}$ on $\partial \Omega$, denoted by $\mathfrak{T}_{q}$. The $\mathfrak{T}_{q}$-closure of a set $E$ will be denoted by $\tilde{E}$. We also need the following notation.

Notation 1.1 (a) For every $x \in \mathbb{R}^{N}$ and every $\beta>0$ put $\rho(x):=\operatorname{dist}(x, \partial \Omega)$ and $\Omega_{\beta}=\{x \in \Omega: \rho(x)<$ $\beta\}, \Omega_{\beta}^{\prime}=\Omega \backslash \bar{\Omega}_{\beta}, \Sigma_{\beta}=\partial \Omega_{\beta}^{\prime}$.

(b) There exists a positive number $\beta_{0}$ such that, for every $x \in \Omega_{\beta_{0}}$ there exists a unique point $\xi \in \partial \Omega$ such that dist $(x, \xi)=\rho(x)$. Put $\sigma(x):=\xi$.

(c) If $Q$ is a $\mathfrak{T}_{q}$-open subset of $\partial \Omega$ and $u \in C(\partial \Omega)$ we denote by $u_{\beta}^{Q}$ the solution of (6) in $\Omega_{\beta}^{\prime}$ with boundary data $h=u \chi_{\Sigma_{\beta}(Q)}$ where $\Sigma_{\beta}(Q)=\left\{x \in \Sigma_{\beta}: \sigma(x) \in Q\right\}$.

Recall that a solution $u$ is moderate if $|u|$ is dominated by a harmonic function. When this is the case, $u$ possesses a boundary trace (denoted by $\operatorname{tr} u$ ) given by a bounded Borel measure. A positive solution $u$ is $\sigma$-moderate if there exists an increasing sequence of moderate solutions $\left\{u_{n}\right\}$ such that $u_{n} \uparrow u$. This notion was introduced by Dynkin and Kuznetsov [5] (see also [6] and [2]).

If $\nu$ is a bounded Borel measure on $\partial \Omega$, the problem

$$
-\Delta u+u^{q}=0 \text { in } \Omega, \quad \operatorname{tr} u=\nu \text { on } \partial \Omega
$$

possesses a (unique) solution if and only if $\nu$ vanishes on sets of $C_{2 / q, q^{\prime}}$-capacity zero, (see [11] and the references therein). The solution is denoted by $u_{\nu}$.

The set of positive solutions of (6) in $\Omega$ will be denoted by $\mathcal{U}(\Omega)$. It is well known that this set is compact in the topology of $C(\Omega)$, i.e., relative to local uniform convergence in $\Omega$. If $u, v \in \mathcal{U}(\Omega)$, we denote by $u \oplus v$ the largest solution dominated by $u+v$.

The first result displays a dichotomy which is the basis for our definition of boundary trace.

Theorem 1.1 Let $u \in \mathcal{U}(\Omega)$ and let $\xi \in \partial \Omega$. If $Q \subset \partial \Omega$ is a $\mathfrak{T}_{q}$-open set and provided the following limit exists, put $L_{Q}=\lim _{\beta \rightarrow 0} \int_{\Sigma_{\beta}(Q)} u d S$. Then,

either $L_{Q}=\infty$ for every $\mathfrak{T}_{q}$-open neighborhood $Q$ of $\xi$,

or there exists a $\mathfrak{T}_{q}$-open neighborhood $Q$ such that $L_{Q}<\infty$.

The first case occurs if and only if, for every $\mathfrak{T}_{q}$-neighborhood $Q$ of $\xi$, 


$$
\int_{A} u^{q} \rho(x) d x=\infty, \quad A=\left(0, \beta_{0}\right) \times Q .
$$

A point $\xi \in \partial \Omega$ is called a singular point of $u$ in the first case and a regular point otherwise. The set of singular points is denoted by $\mathcal{S}(u)$ and its complement in $\partial \Omega$ by $\mathcal{R}(u)$. Our next result provides additional information on the behavior of solutions near the regular boundary set.

Theorem 1.2 Let $u \in \mathcal{U}(\Omega)$. Then $\mathcal{R}(u)$ is $\mathfrak{T}_{q}$-open and there exists a non-negative Borel measure $\mu$ on $\partial \Omega$ possessing the following properties.

(i) For every $\sigma \in \mathcal{R}(u)$ there exist a $\mathfrak{T}_{q}$-open neighborhood $Q$ of $\sigma$ and a moderate solution $w$ such that $\tilde{Q} \subset \mathcal{R}(u), \mu(\tilde{Q})<\infty$ and

$$
u_{\beta}^{Q} \rightarrow w \text { locally uniformly in } \Omega, \quad(\operatorname{tr} w) \chi_{Q}=\mu \chi_{Q} .
$$

(ii) $\quad \mu$ is outer regular relative to $\mathfrak{T}_{q}$ and absolutely continuous relative to $C_{2 / q, q^{\prime}}$ on $\mathfrak{T}_{q}$-open sets on which it is bounded.

Based on these results we define the precise boundary trace of $u$ as the couple $(\mu, \mathcal{S}(u))$. The trace can also be represented by a Borel measure $\nu$ defined as follows. For every Borel set $A \subset \partial \Omega$ :

$$
\nu(A)=\mu(A) \text { if } A \subset \mathcal{R}(u), \quad \nu(A)=\infty \text { otherwise. }
$$

We denote $\operatorname{tr}^{c}=(\mu, \mathcal{S}(u))$ and $\operatorname{tr} u=\nu$.

Let $\nu$ be a positive Borel measure on $\partial \Omega$. We say that $\mu$ is $q$-perfect if:

(i) $\mu$ is outer regular relative to $\mathfrak{T}_{q}$. (ii) $\mu$ is essentially absolutely continuous relative to $C_{2 / q, q^{\prime}}$, i.e., if $Q$ is $\mathfrak{T}_{q^{-}}$-open and $C_{2 / q, q^{\prime}}(A)=0$ then $\nu(Q)=\nu(Q \backslash A)$.

The second property implies that, if $\nu(Q \backslash A)<\infty$ then $\nu(A \cap Q)=0$.

We have the following existence and uniqueness results for the (generalized) boundary value problem (7), where $\operatorname{tr} u=\nu$ is understood as in (10).

Theorem 1.3 Let $\nu$ be a positive Borel measure, possibly unbounded. Then (7) possesses a solution if and only if $\nu$ is q-perfect. When this condition holds, a solution of (7) is given by

$$
U=v \oplus U_{F}, \quad v=\sup \left\{u_{\nu \chi_{Q}}: Q \in \mathcal{F}_{\nu}\right\}
$$

where $\mathcal{F}_{\nu}:=\{Q: Q$ q-open, $\nu(Q)<\infty\}, G:=\bigcup_{\mathcal{F}_{\nu}} Q, F=\partial \Omega \backslash G$ and $U_{F}$ is the maximal solution vanishing on $\partial \Omega \backslash F$.

Theorem 1.4 Let $\nu$ be a q-perfect measure on $\partial \Omega$. Then the solution $U$ of problem (7) defined by (11) is $\sigma$-moderate and it is the maximal solution with boundary trace $\nu$. Furthermore, the solution is unique in the class of $\sigma$-moderate solutions.

For $q_{c} \leq q \leq 2$, results similar to those stated in the last two theorems, were obtained by Dynkin and Kuznetsov [5] and Kuznetsov [6], based on their definition of fine trace. However, by their results, the prescribed trace is attained only up to equivalence, i.e., up to a set of capacity zero. According to the present results, the solution attains precisely the prescribed trace and this applies to all $q \geq q_{c}$. The relation between the Dynkin-Kuznetsov definition and the definition presented here, is not yet clear.

\section{Main ideas of proofs}

We need some additional notation. Let $F \subset \partial \Omega$ be a $\mathfrak{T}_{q}$-closed set and let $U_{F}$ denote the maximal solution vanishing on $\partial \Omega \backslash F$. Then $\inf \left(u, U_{F}\right)$ is a supersolution of (6) and the largest solution dominated 
by it is denoted by $[u]_{F}$.

On the proof of Theorem 1.1. One of the essential features of boundary trace is its local nature. This is used in the present proof, through the following lemmas.

Lemma 2.1 Let $u \in \mathcal{U}$ and let $\left\{\beta_{n}\right\}$ be a sequence converging to zero such that $w=\lim _{n \rightarrow \infty} u_{\beta_{n}}^{Q}$ exists,(see Notation 1.1). Then $[u]_{F} \leq w \leq[u]_{\tilde{Q}}$, for every $\mathfrak{T}_{q}$-closed subset of $Q$.

Lemma 2.2 Let $u \in \mathcal{U}$. Suppose that there exists a $\mathfrak{T}_{q^{-}}$open set $Q \subset \partial \Omega$ and a sequence $\left\{\beta_{n}\right\}$ converging to zero such that $\sup _{n} \int_{\Sigma_{\beta_{n}}(Q)} u d S<\infty$.

Then, for any $\mathfrak{T}_{q}$-closed set $F \stackrel{q}{\subset} Q,[u]_{F}$ is a moderate solution. If $D$ is a $\mathfrak{T}_{q}$-open set such that $\tilde{D} \stackrel{q}{\subset} Q$, there exists a bounded Borel measure $\mu_{D}$ on $\partial \Omega$ such that $\mu_{D}(\partial \Omega \backslash \tilde{D})=0$ and

$$
u(\beta, \cdot) \chi_{D} \rightarrow \mu_{D} \text { weakly relative to } C(\partial \Omega) \text { as } \beta \rightarrow 0 .
$$

The dichotomy stated in the theorem is derived by combining these lemmas.

On the proof of Theorem 1.2. Theorem 1.1 and Lemma 2.2 imply that for every point $\sigma \in \mathcal{R}(u)$ there exists a $\mathfrak{T}_{q}$-open neighborhood $D$ of $\sigma$ and a a bounded Borel measure $\mu_{D}$ on $\partial \Omega$ such that (12) holds. It is not difficult to verify that, if $\sigma, \sigma^{\prime} \in \mathcal{R}(u)$ and $D, D^{\prime}$ are $\mathfrak{T}_{q}$-open neighborhoods related to these points as above then, the corresponding measures $\mu_{D}$ and $\mu_{D^{\prime}}$ are compatible: if $E$ is a Borel subset of $D \cap D^{\prime}$ then $\mu_{D}(E)=\mu_{D^{\prime}}(E)$. The existence of a measure $\mu^{0}$, which vanishes outside $\mathcal{R}(u)$ and satisfies assertion (i) is easily deduced from these facts.

With $\sigma$ and $D$ as above, if $Q$ is a $\mathfrak{T}_{q^{-}}$open set such that $\tilde{Q} \stackrel{q}{\subset} D$ then $[u]_{Q}$ is moderate and $\mu^{0} \chi_{\tilde{Q}}=$ $\mu_{D} \chi_{\tilde{Q}}=\operatorname{tr}[u]_{Q}$. This implies that $\mu^{0}$ is absolutely continuous relative to $C_{2 / q, q^{\prime}}$ and outer regular. On the other hand, if $A$ is a $\mathfrak{T}_{q}$-open set such that $\mu^{0}(A)<\infty$, it follows that on every $\mathfrak{T}_{q^{-}}$-open set $Q$ such that $C_{2 / q, q^{\prime}}(\tilde{Q} \backslash A)=0,(9)$ holds with respect to $\mu^{0}$. Thus $\mu^{0}$ satisfies (ii) in $\mathcal{R}(u)$. The measure $\mu$ defined by

$$
\mu(E)=\inf \left\{\mu_{0}(D): \forall D \text {-open, } E \subset D\right\}
$$

satisfies (ii) on the whole boundary. It is called the $\mathfrak{T}_{q}$-regularization of $\mu_{0}$.

On the proof of Theorem 1.3. The main ingredients in this proof are provided by Theorem 1.2 (ii) and the following lemmas.

Lemma 2.3 Let $F \subset \partial \Omega$ be a q-closed set. Then $\mathcal{S}\left(U_{F}\right)=b_{q}(F)$ where $b_{q}(F)$ denotes the set of $C_{2 / q, q^{\prime}}$ thick points of $F$.

Proof Let $\xi$ be a point on $\partial \Omega$ such that $F$ is $C_{2 / q, q^{\prime}}$-thin at $\xi$. Then there exists a $\mathfrak{T}_{q^{-}}$-open neighborhood $Q$ such that $C_{2 / q, q^{\prime}}(\tilde{Q} \cap F)=0$ and consequently $\left[U_{F}\right]_{Q}=U_{F \cap \tilde{Q}}=0$. Therefore $\xi \in \mathcal{R}\left(U_{F}\right)$.

Conversely, assume that $\sigma \in F \cap \mathcal{R}\left(U_{F}\right)$ and let $Q$ be a $\mathfrak{T}_{q}$-open neighborhood as in Theorem 1.2. Let $D$ be a $q$-open neighborhood of $\sigma$ such that $\tilde{D} \stackrel{q}{\subset} Q$. Then $[u]_{D}$ is moderate and consequently $D \subset \mathcal{R}(u)$. In turn this implies that $C_{2 / q, q^{\prime}}(F \cap D)=0$ and consequently $F$ is $q$-thin at $\xi$.

Lemma 2.4 Let $u \in \mathcal{U}(\Omega)$ and let $\nu:=\operatorname{tr} u$. Put

$$
\mathcal{S}_{0}(u):=\{\xi \in \partial \Omega: \nu(Q \backslash \mathcal{S}(u))=\infty \quad \forall Q: \xi \in Q, Q \text { q-open }\} .
$$

Then $\mathcal{S}(u)=\mathcal{S}_{0}(u) \cup b_{q}(\mathcal{S}(u))$.

The fact that $\mathcal{S}(u) \supset \mathcal{S}_{0}(u) \cup b_{q}(\mathcal{S}(u))$ is straightforward. The opposite inclusion depends on the fact that if $\xi \in \partial \Omega \backslash b_{q}(\mathcal{S}(u))$, there exists a $\mathfrak{T}_{q^{-}}$-open neighborhood $Q$ such that $C_{2 / q, q^{\prime}}(\tilde{Q} \cap \mathcal{S}(u))=0$. Hence $[u]_{Q}=[u]_{Q \backslash \mathcal{S}(u)}$. If $\xi \in \mathcal{S}(u)$ then (8) holds. Therefore, using (10), it is easy to show that $\nu(Q \backslash \mathcal{S}(u))=\infty$. 
Lemma 2.5 Let $u \in \mathcal{U}(\Omega)$ be a $\sigma$-moderate solution and $\left\{u_{n}\right\}$ an increasing sequence of moderate solutions such that $u_{n} \uparrow u$. If $w$ is a moderate solution dominated by $u$ then $\operatorname{tr} w \leq \lim \operatorname{tr} u_{n}$.

Put $\tau:=\operatorname{tr} w$ and $\mu_{0}:=\lim \operatorname{tr} u_{n}$. It is sufficient to show that $\tau(K) \leq \mu_{0}(K)$ for every compact set $K \subset \partial \Omega$ such that $\mu_{0}(K)<\infty$ and $C_{2 / q, q^{\prime}}(K)>0$. It can be shown that, under these assumptions: (a) There exists a $\mathfrak{T}_{q^{-}}$open set $Q$ such that $C_{2 / q, q^{\prime}}(K \backslash Q)=0$ and $\mu_{0}(Q)<\infty$. (b) If $F$ is a $\mathfrak{T}_{q^{-}}$-closed subset of $Q$ then $\nu(F) \leq \mu_{0}(Q)$.

These facts imply in a straightforward manner that $\tau(K) \leq \mu_{0}(K)$.

Combining Lemma 2.3, Lemma 2.4 and Theorem 1.2 (ii) one can verify that the existence of a solution $u \in \mathcal{U}(\Omega)$ such that $\nu=\operatorname{tr} u$ implies that $\nu$ is $q$-perfect.

On the other hand, we observe that the solution $U$ defined by (11) is $\sigma$-moderate. This is based on the fact that $U_{F}$ is $\sigma$-moderate, which (for $F$ compact) was established by the authors in [12] and it remains valid when $F$ is $\mathfrak{T}_{q^{-}}$-closed. Using this fact and Lemma 2.5 it can be shown that, if $\nu$ is $q$-perfect then $\operatorname{tr} U=\nu$.

On the proof of Theorem 1.4. The uniqueness result is based on,

Lemma 2.6 Let $u \in \mathcal{U}(\Omega)$ be a $\sigma$-moderate solution and $\left\{u_{n}\right\}$ an increasing sequence of moderate solutions such that $u_{n} \uparrow u$. Put $\nu_{0}=\lim \operatorname{tr} u_{n}$ and let $\nu$ be the $\mathfrak{T}_{q}$-regularization of $\nu_{0}$. Then $\nu$ is the precise boundary trace of $u$. In particular it is independent of the choice of the sequence $\left\{u_{n}\right\}$.

Let $\left\{u_{n}\right\}$ and $\left\{w_{n}\right\}$ be two increasing sequences of moderate solutions converging to $u$. Then Lemma 2.5 implies that $\lim \operatorname{tr} u_{n}=\lim \operatorname{tr} w_{n}$. Thus $\nu$ is independent of the choice of the sequence and it is not difficult to verify that $\operatorname{tr} u=\nu$.

Clearly, Lemma 2.6 implies that, if $u, v$ are $\sigma$-moderate solutions and $u \leq v$ then $\operatorname{tr} u \leq \operatorname{tr} v$. Hence the uniqueness result. In addition, if $u \in \mathcal{U}(\Omega)$ and $\nu=\operatorname{tr} u$ then the solution $v$ defined as in Theorem 1.3 is uniquely determined by $\nu$ and therefore by $u$. It can be sown that $u \ominus v$ (= smallest solution dominating $u-v$ ) vanishes on $\mathcal{R}(u)$. Therefore $u \ominus v \leq U_{F}$ where $F=\mathcal{S}(u)$. Hence $U=v \oplus U_{F}$ is the maximal solution with trace $\nu$.

Acknowledgment. Both authors were partially sponsored by an EC grant through the RTN Program Front-Singularities, HPRN-CT-2002-00274 and by the French-Israeli cooperation program through grant No. 3-1352. The first author (MM) also wishes to acknowledge the support of the Israeli Science Foundation through grant No. 145-05.

\section{References}

[1] Adams D. R. and Hedberg L. I., Function spaces and potential theory, Grundlehren Math. Wissen. 314, Springer (1996).

[2] Dynkin E. B. Diffusions, Superdiffusions and Partial Differential Equations, American Math. Soc., Providence, Rhode Island, Colloquium Publications 50, 2002.

[3] Dynkin E. B. Superdiffusions and Positive Solutions of Nonlinear Partial Differential Equations, American Math. Soc., Providence, Rhode Island, Colloquium Publications 34, 2004.

[4] Dynkin E. B. and Kuznetsov S. E. Superdiffusions and removable singularities for quasilinear partial differential equations, Comm. Pure Appl. Math. 49, 125-176 (1996).

[5] Dynkin E. B. and Kuznetsov S. E. Fine topology and fine trace on the boundary associated with a class of quasilinear differential equations, Comm. Pure Appl. Math. 51, 897-936 (1998). 
[6] Kuznetsov S. E. $\sigma$-moderate solutions of $L u=u^{\alpha}$ and fine trace on the boundary, C.R. Acad. Sc. Serie I 326, 1189-1194 (1998).

[7] Legall J. F., The Brownian snake and solutions of $\Delta u=u^{2}$ in a domain, Probab. Th. Rel. Fields 102, 393-432 (1995).

[8] Legall J. F., Spatial branching processes, random snakes and partial differential equations, Birkhäuser, Basel/Boston/Berlin, 1999.

[9] Marcus M. and Véron L., The boundary trace of positive solutions of semilinear elliptic equations: the subcritical case, Arch. Rat. Mech. Anal. 144, 201-231 (1998).

[10] Marcus M. and Véron L., The boundary trace of positive solutions of semilinear elliptic equations: the supercritical case, J. Math. Pures Appl. 77, 481-524 (1998).

[11] Marcus M. and Véron L., Removable singularities and boundary trace, J. Math. Pures Appl. 80, 879-900 (2000).

[12] Marcus M. and Véron L., Capacitary estimates of positive solutions of semilinear elliptic equations with absorption, J.European Math. Soc. 6, 483-527 (2004). (2004).

[13] Mselati B., Classification and probabilistic representation of the positive solutions of a semilinear elliptic equation, Mem. Am. Math. Soc. 168 (2004). 